\title{
"- It would be possible to do a lengthy dialectical number on this;"
}

\author{
Wayne C. Myrvold \\ Department of Philosophy \\ The University of Western Ontario \\ wmyrvold@uwo.ca
}

December 1, 2019

\begin{abstract}
Philosophers have, it seems, been beguiled by contingencies of the evolution of scientific language. These contingencies can obscure the nature of theoretical shifts. Retention of a term can obscure a radical theoretical shift, and abandonment of a term can obscure continuity of theory. In this paper, I consider the cases of caloric and the luminiferous ether, both of which are often taken to be unproblematic cases of theoretical entities abandoned by subsequent theories. I invite the reader to consider what we now might be saying were those terms retained, and argue that this sheds light on the nature of the theoretical shifts involved.
\end{abstract}

\section{Introduction}

In the controversies between scientific realists and anti-realists, it is common for anti-realists to enlist episodes in which theoretical posits once accepted by a scientific community came to be rejected, in service of a meta-inductive argument for the conclusion that we should expect some, or most, or perhaps even all of what is currently accepted to suffer the same fate. Realists, on the other hand, tend to stress continuity across theory changes. In all of these discussions, certain things tend to be taken for granted by both sides. Among these are: 
nineteenth-century theories of electromagnetism posited the existence of an electromagnetic ether, which was abandoned in the twentiethcentury, and heat was once thought to consist of a fluid, called caloric, now regarded as nonexistent.

About discussions of this sort, Howard Stein commented, in his "Yes, but... Some Skeptical Remarks on Realism and Anti-Realism,"

For my part, I throw up my hands at this: Why should we say that the old term "ether" failed to "refer"? — and that the old term "atom" did "refer"? Why, that is, except for the superficial reason that the word "atom" is still used in text-books, the word "ether" not? - It would be possible to do a lengthy dialectical number on this; but in brief: our own physics teaches us that there is nothing that has all the properties posited by nineteenth-century physicists for the ether or for atoms; but that, on the other hand, in both instances, rather important parts of the nineteenth-century theories are correct. For instance, so far as Poincaré's conviction that momentum conservation must hold among the "particles" taken by themselves is concerned, it is established now beyond a doubt that ordinary bodies do exchange momentum with "the ether" i.e., with the electromagnetic field; and even that this field has to be regarded as the seat of a distribution of mass, and as participating in gravitational interactions. The two cases - that of the ether and that of atoms - are, in my view, so similar, that the radical distinction made between them by the referential realists confirms in me the antecedent suspicion that this concern for reference - and associated with it, another Quinean motif, the concern for what is called the "ontology" of theories - is a distraction from what really matters (Stein, 1989, 56-57).

I share Stein's dismay, and I suspect that philosophers - myself includedhave been beguiled by contingencies of the evolution of scientific language.

I would dearly like to know what Stein would have written, had he written that lengthy dialectical number. What I offer here is a brief and humble dialectical number of my own. I find it useful to consider what we might now be saying if certain contingent and some- 
what arbitrary choices of language had gone otherwise. ${ }^{1}$ There are, I claim, nearby possible worlds in which the word caloric was adopted by proponents of the kinetic theory of heat, and in which the word ether survived the advent of special relativity and continues to be in use today. Moreover, these are nearby possible worlds in which the shifts in scientific thought, as far as substantive content of what was accepted and what was rejected, occurred in exactly the same way as they did in the actual world. In these alternate worlds, the content of currently accepted science is exactly what it is in our world; these worlds differ from ours only in terminological choices. Readers may well at this point be skeptical of the claim that these are nearby possible worlds. I urge such readers to read on; the case for this claim will be made in the following.

Moreover, there is something to be said for the terminological choices made by our counterfactual counterparts, as they shed light on the nature of the shifts that occurred. These shifts are traditionally expressed in something like the following way.

1. Heat was formerly thought to be a substance, called caloric, which flows from warmer bodies to cooler. The kinetic theory of heat does away with caloric, locating heat in the kinetic energy of molecules.

2. During the 19th century, electric and magnetic fields were thought of as disturbances in a subtle, all-pervasive medium, called the ether. Einstein, in 1905, showed the ether to be superfluous, and it is now thought to be nonexistent.

If such well-established theoretical entities, central to well-established scientific theories, can be abandoned, ask the proponents of the pessimistic meta-induction, what hope do we have that the theoretical entities of our current theories will survive? A common realist response is to argue that, contrary to what might have appeared at the time, the posited entities were not essential to the empirical success of those theories.

Common to the realist and anti-realist is the shared assumption

\footnotetext{
${ }^{1} \mathrm{My}$ goal is not merely to emphasize the contingency of terminological shifts. This point has made been before; see, in particular, Stanford (2015). My goal is, rather, to argue that, in the two cases examined here, involving the abandonment of the terms "ether" and "caloric," the terminological shift has obscured the nature of the theoretical shift, and to urge that consideration of how one might express things had these terms not been abandoned affords a clearer view of what transpired.
} 
that, according to current science, caloric and the luminiferous ether do not exist. This, in my opinion, concedes too much to the pessimist. It also distracts from what is, for me, the most interesting issue: getting clear about what we do, and do not, agree about with our predecessors. In place of the traditional expression of these shifts, I offer the following.

1. Though we now know that caloric is not conserved, Carnot's assumption that it is conserved did not impair his reasoning about it, because that reasoning had to do with circumstances in which it is conserved.

2. What Einstein and Lorentz disagreed about was whether the luminiferous ether has the sort of structure that permits one to speak of motion with respect to it. According to Einstein, though the properties of the ether may vary from point to point, locally it is sufficiently homogeneous that talk of velocity with respect to the ether does not make sense.

If these seem quixotic at the moment, read on; I will explain. But first, a few words about what I am not doing in this paper.

There is a distressing tendency in philosophy of science to draw sweeping conclusions from consideration of a small number of historical examples carefully selected to serve the author's purpose. I am not engaged in that activity. In particular, I am not attempting to make predictions, based on an inductive argument, about what elements of contemporary science will survive into the next century. If one were to attempt seriously the project of meta-induction, and to try to estimate, via enumerative induction, the percentage of currently accepted propositions that will survive one hundred or two hundred years of future scientific developments, one would first need a historical data-set that could be regarded as an unbiased sample of previously accepted propositions. I have no idea how one would go about constructing an appropriate data-set, but it should be clear that the method of selective anecdote that is too common in the philosophy of science literature has no probative value whatsoever. An appearance of support can be produced for anything one desires, if one is allowed to be selective about the evidence. The morals I draw will not be of the meta-inductive sort.

I am also not making a merely Quinean point that "any statement can be held true come what may, if we make drastic enough adjustments elsewhere in the system." If no qualifications (such as 
a requirement that one's behaviour be consistent with an honest and sincere endeavour to learn about the world) are introduced, then, certainly, one can do all sorts of things, including insisting on blatant falsehoods in the face of evidence of their falsity. Neither Quine nor anyone else has offered an argument that a serious researcher engaged in a project of understanding the world can hold any statement true, come what may. My aim is higher. I will argue that the terminological choices to be considered in this paper could have been adopted by a community of serious researchers engaged in a project of trying to understand what there is in the world and what it is like. And, indeed, each of the terminological choices that I will be describing has been advocated by a serious researcher; it just so happens that the proposed terminology did not catch on.

\section{The luminiferous ether}

From the 17th to the 19th centuries, the wave theory of light was associated with the postulation of a subtle medium, the luminiferous ether, thought of as the bearer of light waves. In the mid-nineteenth century this was joined by an electromagnetic ether, the seat of electric and magnetic fields. Faraday's discovery in 1845 that the plane of polarization of light can be rotated by a magnetic field requires that there be interaction between magnetic fields and light waves (Faraday, 1846).

The notion of field that is suggested by Faraday's work was developed by Maxwell. This occurs in three stages. In the first, "Faraday's Lines of Force" (1858), Maxwell develops an analogy between the equations of electrostatics and the flow of an incompressible fluid. At this stage he explicitly disavows formulating a physical theory; the role of these analogies are to "bring before the mind, in a convenient and manageable form, those mathematical ideas which are necessary to the study of the phenomena of electricity" (Maxwell 1858, 29; Niven 1890, 157). In the second, "On Physical Lines of Force," (1861; 1862), following the lead of Kelvin (Thomson 1847), who exhibited an analogy between equations of electromagnetism and equations of equilibrium of an elastic solid, Maxwell presents a mechanical model on which magnetic fields are associated with molecular vortices in some medium, with the magnetic field vectors pointing along the axes of the vortices. The vortices resist compression and hence the collection of them acts 
as an elastic solid. Small, spherical particles are introduced as idle wheels between neighbouring vortices; the motion of these particles constitute an electric current in conductors. In dielectrics (considered in Part III), these particles never venture far from their equilibrium positions; under stress, they can be displaced slightly. When the stress is relaxed, the electric particles return to their original position, and this movement of electricity (the displacement current) is treated as exactly equivalent to a current in conductors, and, crucially, generates a magnetic field, just as a conduction current does. Maxwell concludes that transverse vibrations can propagate through the elastic medium, and estimates their velocity as equal to the ratio between electromagnetic and electrostatic units (which has dimension length/time), recently measured by Kohlrausch and Weber as 310,740,000,000 mm. per second. This agrees so closely with the speed of light in air, measured by Fizeau, that Maxwell declares that "we can scarcely avoid the inference that light consists in the transverse undulations of the same medium which is the cause of electric and magnetic phenomena" (Maxwell 1862, 22; Niven 1890, 500). ${ }^{2}$

At this stage Maxwell takes the elastic medium to be a reality, though he is not committed to the details of his somewhat baroque mechanical model (he is confident, though, that magnetic fields are associated with some sort of rotation of the medium). In his mature theory, presented in "A Dynamical Theory of the Electromagnetic Field" (1865) and his monumental Treatise on Electricity and Magnetism (1873a,b), he eschews mechanical models, presuming only that in the space surrounding electric and magnetic bodies there is matter in motion, to which the methods of Lagrangian mechanics can be applied. What we know about the electromagnetic field is the energy associated with various states of the field. The Treatise concludes,

whenever energy is transmitted from one body to another in time, there must be a medium or substance in which the energy exists after it leaves one body and before it reaches the other, for energy, as Torricelli remarked, 'is a

\footnotetext{
${ }^{2}$ Maxwell's calculation, in 1862 , was based on consideration of his molecular-vortex model, and involved some somewhat arbitrary assumptions about parameters of that model. His conclusion that the velocity of transverse vibrations is equal to the ratio between electromagnetic and electrostatic units should be regarded as an order-of-magnitude estimate (see Siegel 1991, Ch. 5, for details). The now-familiar derivation of a wave equation for the fields (Maxwell considers the magnetic field, but essentially the same derivation goes through for the electric), is found in Part VI of Maxwell (1865).
} 
quintessence of so subtile a nature that it cannot be contained in any vessel except the most inmost substance of material things.' Hence all these theories lead to a conception of a medium in which the propagation takes place, and if we admit this medium as an hypothesis, I think it ought to occupy a prominent place in our investigations, and that we ought to endeavour to construct a mental representation of all the details of its action, and this has been my constant aim in this treatise (Maxwell, 1873b, 438).

Those of us whose introduction to classical electromagnetic theory occurred in either the 20th or 21st centuries, through the medium of standard textbooks, are familiar with a picture according to which charges are associated with ponderable matter, and are distinct from electromagnetic fields. ${ }^{3}$ Currents are moving charges, on this picture, and charges and currents bear a two-sided relation to the fields: they act as sources of fields, and are acted upon by the fields via the Lorentz force law. On this picture, the free-space fields $\mathbf{E}$ and $\mathbf{B}$ are primary, and the "macroscopic" fields, $\mathbf{D}$ and $\mathbf{H}$, are derived as averages over regions large enough to contain a great many molecules, with which may be associated electrical or magnetic moments.

This textbook classical electromagnetic theory, with a dualistic ontology of charged matter and electromagnetic fields, and a delimitation of scope according to which it is not the business of the theory to explain the structure or stability of ponderable matter (so that charges and currents as sources may be taken as exogenously given), is a 20 th century construction from 19 th century materials. It does not correspond to Maxwell's conception of the theory, or to that of his immediate successors.

Though it is clear that the now-familiar textbook picture is not Maxwell's view, what, precisely, he does think is not easy to glean from his mature writings. Poincaré, in the introduction to his lectures on electricity and optics, says, in regards to Maxwell's Treatise,

One of the French scientists who has probed Maxwell's work the most deeply said to me one day, 'I understand everything in this book, except what is meant by a charged sphere' (quoted in translation by Bromberg 1968, 142, from

\footnotetext{
${ }^{3}$ For our purposes, we may take Jackson (1962) and subsequent editions as representative of the textbook tradition referred to. This has not only been a widely used text; its method of exposition has influenced other textbooks.
} 
Poincaré 1890, xvi-xvii). ${ }^{4}$

A modern reader may well share this puzzlement when encountering locutions such as "free positive electricity ... arises from the electrification of the different parts of the field not neutralizing each other," (Maxwell 1865, 485; Niven 1890, 561) and, in $\S 111$ of the Treatise, "According to this theory, all electrification is the residual effect of the polarization of the dielectric," (Maxwell, 1873a, 133) which becomes, in the second edition, "all charge is the residual effect of the polarization of the dielectric" (Maxwell, 1881, 155). ${ }^{5}$

On Maxwell's theory of electric displacement, an electric field in the interior of a dielectric medium is realized as a strain in the medium, which produces displacements of positive and negative electricity which, in any region in which the displacement is constant, average to zero. Where the displacement vector field has a nonzero divergence, there will be within any small region a net charge, as more charge is displaced out of it on one side than into on the other.

At the boundary between a dielectric and a conductor, there will be a discontinuity in the displacement vector, and a net charge at the surface. Crucially, this remains true when the boundary is a boundary between ponderable matter and what we would think of as empty space. What we think of as a vacuum, Maxwell regards as filled with a polarizable dielectric medium, the ether. ${ }^{6}$

It was Lorentz who decisively located charge in "ponderable matter perfectly permeable to the ether and able to move without communicating the least motion to the ether" $(1892,70) .^{7}$ This is not only a bold move, it is one of questionable consistency with other ideas. Though the ether interacts with ponderable matter, exchanging energy with it, the latter communicates no motion to the ether. As Stein $(1978,383)$ notes, Lorentz did not present this move as "revolutionary." Nonetheless, Stein's characterization of the move is apt.

What in effect he [Lorentz] did deserves comparison with

\footnotetext{
${ }^{4}$ Un des savants français qui ont le plus approfondi l'oeuvre de Maxwell me disait un jour : «Je comprends tout dans son livre, excepté ce que c'est qu'une boule électrisé. »

${ }^{5}$ Though the second edition of the Treatise was published posthumously, the first nine chapters are based on Maxwell's own revisions.

${ }^{6}$ See Bromberg (1968), Buchwald (1985, Ch. 3), Siegel (1991, 150-151), Darrigol (2000, §4.4.8) for discussions of this point.

${ }^{7}$ See Hirosige (1969) for a detailed account of the development of this conception in Lorentz' work. The first step towards this conception is taken already in his dissertation, (Lorentz, 1875), but it is not fully realized until 1895.
} 
such other cuttings of Gordian knots as that by Einstein in his 1905 quantum paper and that by Bohr in his 1913 papers on the constitution of atoms and molecules: in his quiet way, he had the boldness and the insight to combine those parts of existing physical theory that could lead to definite and interesting results, while ignoring - as in the case of Einstein and Bohr, tentatively and in the spirit of heuristic inquiry - what he saw no way to incorporate consistently (Stein, 1978, 383).

Perhaps, after all, we should recognize Lorentz as a quiet revolutionary (384).

In Lorentz' version of electromagnetism, electromagnetic waves are local disturbances in a stationary ether. The velocity implicit in Maxwell's equations, which is the velocity of freely propagating electromagnetic waves, is velocity with respect to this ether, as is the velocity that appears in the Lorentz force law.

There is a substantial lacuna in Maxwell's treatment of optical phenomena, that may be surprising to modern readers. His treatment of light is restricted to wave propagation in homogeneous media. Neither in the "Dynamical Theory" nor in the Treatise does he deal with reflection and refraction of light at the boundary of two media with differing indices of refraction. Thus, one will find in Maxwell no electromagnetic explanation of Fresnel's laws of reflection and refraction, which have become a centerpiece of the discussions surrounding structural realism. The stumbling block was the question of boundary conditions to impose at the interface between the two media. ${ }^{8}$ This problem was dealt with by Lorentz in his doctoral dissertation (1875; 1997). Lorentz was inspired by a remark made in a footnote in a paper of Helmholtz, in which Helmholtz announced (without giving details) that the laws of reflection and refraction hold at the boundary of two insulating media when either the electric or magnetic polarisability of the two media are the same (Helmholtz 1870, 68; 1882, 559). It was also dealt with by Fitzgerald (1880), who adopted boundary conditions from MacCullagh (1846). ${ }^{9}$ In his referee report on a draft of Fitzgerald's paper (Maxwell, 1879), Maxwell expressed dissatisfaction with Fitzgerald's treatment of boundary conditions, urging the author

\footnotetext{
${ }^{8}$ See Maxwell's letter to Stokes, 15 October 1864, in Harman (1995, 186-188).
}

${ }^{9}$ See Stein (1981, 310-315) and Saatsi (2005) for discussion. 
to be more explicit about the physical assumptions made. ${ }^{10}$ Sadly, Maxwell did not live to comment on the final version of Fitzgerald's paper.

It follows from Maxwell's equations ${ }^{11}$ that, at a boundary surface between two dielectrics, on which there may or may not be a surface charge density and surface current density, the components of $\mathbf{E}$ tangent to the surface, and the component of $\mathbf{B}$ normal to the surface, must be continuous. If there is surface charge at the boundary, there is a discontinuity in the component of $\mathbf{D}$ perpendicular to the surface as it crosses the boundary, proportional to the surface charge density at that point. If there are surface currents, there is a discontinuity in the tangential components of $\mathbf{H}$ at the boundary, equal in magnitude to the magnitude of the surface current density (see Appendix for proofs of these assertions). In his dissertation, Lorentz imposes boundary conditions that are equivalent to the continuity of the component of D perpendicular to the surface and of the tangential component of $\mathbf{H}$ at the boundary. ${ }^{12}$ These conditions are equivalent to the absence of free surface charges or currents at the boundary. The modern textbook tradition follows Lorentz in this (see Jackson 1962, §7.5).

From the point of view adopted by Lorentz and the textbook tradition, which locates charges in ponderable matter rather than in the electromagnetic field, one can simply stipulate that the situation at hand is one in which surface charges are absent, and continuity of the perpendicular component of $\mathbf{D}$ at the boundary follows from this assumption. On Maxwell's view of charge, continuity of $\mathbf{D}$ at the boundary cannot be thought of as a consequence of an absence of charge at the boundary, which one can impose as an exogenous constraint on the problem. Rather, because a surface charge at the boundary is nothing other than a discontinuity in the electric displacement $\mathbf{D}$ at that loca-

\footnotetext{
10 "his statement of the boundary conditions. . . can only be interpreted by working backwards from his results."

${ }^{11}$ In this paper, the term Maxwell's equations is used according in conformity with 20th-century textbook tradition, for the familiar set of four equations relating electrical and magnetic fields to each other and to source charges and currents. These equations are, unsurprisingly, not those of Maxwell, who summarized the basic equations of electromagnetism in a set of 12 equations in which the electric and magnetic potentials figure prominently (see Maxwell 1873b, Ch. IX). The first appearance of the basic equations of Maxwellian electromagnetism written in terms of the fields directly occurs in Heaviside's "Electromagnetic Induction and its Propagation," published in 1885; see Heaviside (1892, Art. XXX).

${ }^{12}$ See Hirosige $(1969,168)$.
} 
tion, assuming absence of surface charge is precisely the assumption of continuity at the boundary. This may help to explain why Maxwell was at a loss to formulate physically well-motivated boundary conditions at the interface between two dielectrics that would suffice to obtain laws of reflection and refraction. The upshot of this is that it is misleading to say that Fresnel's laws of reflection and refraction follow from Maxwell's theory. They follow from Lorentz's transfigured version of Maxwell's theory.

The next step, from Lorentz's reformulation, towards the modern view, is well-known. Einstein, dissatisfied with the fact that, in the analysis of certain phenomena, such as induction of current in a conductor moving with respect to a magnet, there is an asymmetry in the theoretical treatment that does not appear in the phenomena-the theoretical treatment differs, depending on which bodies are moving with respect to the ether, whereas the observable phenomena depend only on the relative motion of ponderable bodies - reformulates electromagnetic theory in a way that makes no use of the motion of bodies with respect to the ether, and declares,

The introduction of a "light ether" will prove superfluous, inasmuch as in accordance with the concept to be developed here, no "space at absolute rest" endowed with special properties will be introduced, nor will a velocity vector be assigned to a point of empty space at which electromagnetic processes are taking place (Einstein 1989, 141, from Einstein 1905, 892).

This is the first clear articulation of the modern view of electromagnetic fields as self-subsistent in otherwise empty space. It would not have been possible on Maxwell's theory, as, on that theory, to do away with the elastic medium that is the seat of electromagnetic fields would be to do away with the charges on the surface of a charged conductor.

The transition from ether-based theories to a view on which electromagnetism deals with free-standing fields in otherwise empty space is, in the literature on structural realism, referred to in a way that strikes me as misleading. It is almost universally referred to as the "Fresnel-Maxwell" transition. This locution stems from Worrall.

Fresnel's wave theory itself was soon replaced by Maxwell's electromagnetic theory. Maxwell, as is well known, strove manfully to give an account of the electromagnetic field in terms of some underlying mechanical medium; but his 
attempts and those of others failed and it came to be accepted that the electromagnetic field is a primitive. So again a fundamental change in the accepted account of the basic structure of light seems to have occurred - instead of vibrations carried through an elastic medium, it became a series of wave-like changes in a disembodied electromagnetic field. A mechanical vibration and an electric ('displacement') current are surely radically different sorts of thing (Worrall, 1989, 108).

Maxwell's theory and then the General Theory of Relativity entail that there is just no such thing as Fresnel's elastic optical ether. According to these later theories, light "in fact" consists of vibrations of the electromagnetic field, a field which is "sui generis", explicitly not a manifestation of the contortions of some underlying material medium. (As is well known, Maxwell himself tried hard to produce a "mechanical model" for the field - that is, to explain the field in terms of some underlying material medium. But his failure in this attempt, and the failure of his contemporaries and successors, led to the acceptance of what might be called the "mature" version of Maxwell's theory - a theory that sees the field as a "primitive" part of the furniture of the universe.) (Worrall, 1994, 335)

Now, although there is a sense in which what emerged from the work of Lorentz and then Einstein was a maturation of Maxwell's theory, to say that it is Maxwell's theory that entails that there is no elastic optical ether is to disregard the transformation of the theory, in Lorentz' hands, that was required to pave the way for Einstein's declaration that the ether is superfluous, a quarter-century after Maxwell's untimely death. And to refer to a conception of electromagnetic fields that was first articulated in the 20th century, and which is completely alien to Maxwell's conception, as Maxwell's theory, in articles that will be read by students who are unfamiliar with the actual history but in whom we are trying to inculcate a respect for actual history, strikes me as a poor choice.

The story, as told in the scientific realism literature, also omits the further development of Einstein's thinking. As we have seen, in 1905 Einstein declared the ether to be superfluous. By 1920 he was expressing things differently. In an address delivered in May, 1920, at 
the University of Leiden (with Lorentz present), Einstein says,

He [Lorentz] achieved this, the most important advance in the theory of electricity since Maxwell, by taking from ether its mechanical, and from matter its electromagnetic qualities. As in empty space, so too in the interior of material bodies, the ether, and not matter viewed atomistically, was exclusively the seat of electromagnetic fields. According to Lorentz the elementary particles of matter alone are capable of carrying out movements; their electromagnetic activity is confined to the carrying of electric charges. Thus Lorentz succeeded in reducing all electromagnetic happenings to Maxwell's equations in free space.

As to the mechanical nature of the ether, it may be said of it, in a somewhat playful spirit, that immobility is the only mechanical property of which it has not been deprived by H. A. Lorentz. It may be added that the whole change in the conception of the ether which the special theory of relativity has brought about, consisted in taking away from the ether its last mechanical property, namely, its immobility (Einstein 1922, 10-11, from Einstein 1920, 7).

On this way of expressing the matter, the transition to special relativity consisted in the realization that the remaining mechanical property ascribed to it, immobility, actually played no role in electromagnetic theory.

The next position which it is possible to take up in the face of this state of things [that is, the lack of an empirical distinction between rest and uniform motion with respect to the ether] appeared to be the following. The ether does not exist at all. The electromagnetic fields are not states of a medium, and are not bound down to any bearer, but they are independent realities which are not reducible to anything else, exactly like the atoms of ponderable matter (Einstein 1922, 12, from Einstein 1920, 8).

This, of course, is Einstein's view as of 1905. But it is not his final view.

More careful reflection teaches us, however, that the special theory of relativity does not compel us to deny ether. We may assume the existence of an ether; only we must give up 
ascribing a definite state of motion to it, i.e., we must take from it the last mechanical characteristic which Lorentz had left it (Einstein 1922, 13, from Einstein 1920, 9).

Later, Einstein wrote, "Today his [Lorentz's] discovery may be expressed as follows: physical space and the ether are only different terms for the same thing; fields are physical states of space" (Einstein 1954, 281, from Einstein 1934).

Einstein is right about this, I think, though Lorentz himself did not agree. The move made by Lorentz, of locating charges and currents in ponderable matter and assigning to the ether no other role than as seat of electromagnetic fields, does make "ether" merely another word for space devoid of ponderable matter. The remaining point of disagreement between them had to do with the structure of this ether - does it possess the sort of structure that permits one to speak unambiguously of motion with respect to it?

To describe, as is common in the historical literature on this topic, Lorentz as an ether theorist and to take Einstein as having shown the ether to be superfluous obscures both the revolutionary move made by Lorentz and the continuity between Einsteinian and Lorentzian electrodynamics. To describe, as is common in the literature on structural realism, the Einstein 1905 view as a "mature" version of Maxwell's theory also obscures the distance between Maxwell and Lorentz on this matter. Things become clearer if we describe the progression of ideas as Einstein recommends. Lorentz strips the ether of all "mechanical" properties besides providing a standard of rest and motion; Einstein showed that it doesn't even play that role, as (contrary to first appearances), state of motion with respect to the ether plays no essential role in electromagnetic theory.

\section{Caloric}

Among the entries in Lavoisier's Table of Simple Substances in his Traité Élémentaire de Chimie is calorique, or caloric, which he regards as identical to what other writers had referred to variously as heat, principle of heat, fire, igneous fluid, or matter of fire and heat (Lavoisier 1789, 192; 1790, 175). It is difficult, Lavoisier says, to conceive of the effects of heat on matter "without admitting them as the effects of a real and material substance, or a very subtle fluid, which, insinuating itself between the particles of bodies, separates them from 
each other; and, even supposing the existence of this fluid to be hypothetical, we shall see in the following that it explains all of the phenomena of nature in a very satisfactory manner" (1790, 4, from Lavoisier 1789,4$).{ }^{13}$

On the traditional story, a rival theory, the kinetic theory of heat, according to which heat is not a substance contained in hot bodies but rather, subsists in the kinetic energy of molecules making up those bodies, gradually gained ascendance. Instrumental in the transition were Count Rumford's experiments concerning the generation of heat by friction. Rumford argued that what his experiments showed was that there was no limit to the heat that might be obtained from a given body via friction. Also instrumental in the shift in the conception of the nature of heat were Joule's experiments, in the 1840s, on the mechanical equivalent of heat. Expenditures of equal amounts of mechanical energy produce equal amounts of heat, as measured by, say, the increase of temperature produced in a given quantity of water. This permits heat to be measured in terms of its mechanical equivalent; we would now say that both heat and work can be measured in terms of energy. The interconvertibility of heat and work became a cornerstone of the science of thermodynamics.

The second cornerstone of the developing science of thermodynamics was Carnot's analysis of the efficiency of heat engines. In the familiar textbook presentation, a reversible engine is considered that operates between two heat reservoirs. The engine is operated in a cycle that involves extraction of an amount of heat $Q_{1}$ from the hotter reservoir, part of which is converted into work $W$, with the remainder, $Q_{2}$, deposited into the colder reservoir. Conservation of energy requires

$$
Q_{1}=W+Q_{2}
$$

We can define the efficiency of the engine as the proportion of the heat extracted from the hotter reservoir that is converted into work.

$$
\eta=\frac{W}{Q_{1}}=\frac{Q_{1}-Q_{2}}{Q_{1}} .
$$

${ }^{13}$ It should be noted that Lavoisier was not dogmatic about the nature of caloric; "we are not obliged to suppose this to be a real substance; it being sufficient, as will be more clear in the following, that it be considered as the repulsive cause, whatever that may be, which separates the particles of matter from each other, so that we are still at liberty to investigate its effects in an abstract and mathematical manner" (1790, 5-6, from Lavoisier $1789,5-6)$. 
Those who are familiar with this presentation may be puzzled to learn that Carnot regarded heat as a conserved fluid, and took it as axiomatic that the same amount of heat is deposited into the colder reservoir as is extracted from the hotter. Kelvin himself, at the time he wrote the paper in which he first introduced an absolute temperature scale, shared this assumption.

In the present state of science no operation is known by which heat can be absorbed, without either elevating the temperature of matter, or becoming latent and producing some alteration in the physical condition of the body into which it is absorbed; and conversion of heat (or caloric) into mechanical effect is probably impossible*, certainly undiscovered (Thomson 1848, 68, in Thomson 1882, 102. See also Thomson 1849, in Thomson 1882, 117).

Occurring in an article that contains an analysis of the Carnot cycle, the assertion that there is no known process by which heat is converted to mechanical effect is likely to seem puzzling to modern readers.

The solution to the puzzle can be found by considering how quantity of heat is to be determined. The now-standard analysis is predicated on taking the quantities of heat $Q_{1}$ and $Q_{2}$ to be measured by their mechanical equivalents, which, as Joule demonstrated to many researchers' satisfaction, agrees with heat as measured by calorimetric methods. That is, $Q_{1}$ and $Q_{2}$ are the amounts of energy transferred in the two heat exchanges of the engine with the reservoirs.

That this is the appropriate measure of quantity of caloric is not something that ought to be taken for granted, in the context of Carnot's account of the motive power of heat. According to him, the motive power of a given quantity of caloric is a function of temperature. In a famous analogy, he compares the motive power of heat to the motive power of water, which is a function of height.

The motive power of a waterfall depends on the height and the quantity of the liquid; the motive power of heat depends also on quantity of caloric used, and on what may be termed, on what in fact we will call, the height of its fall [la hauteur de sa chute], that is to say, the difference of temperature of the bodies between which the exchange of caloric is made. (Carnot 1890, 61, from Carnot 1824, 28; see also Magie 1899, 16).

Though the concept would not be fully developed for another decade 
or so, we can see that Carnot here has a glimmering of the concept we now call potential energy. When water falls from a height, turning a wheel, the quantity of water is not diminished, but some of its potential energy is converted into mechanical energy. Carnot sees the action of heat to be analogous; in a heat engine, a quantity of caloric is taken from a higher temperature to a lower temperature, and it thereby loses some of its motive power, as some of this motive power is converted into mechanical effect.

We thus see that, on this way of viewing things, there is a potential ambiguity in the phrase "quantity of heat." When heat passes from one body to another, a quantity of caloric is exchanged, and with it a temperature-dependent quantity of energy. Either of these might be called "quantity of heat." Let us see what follows from taking seriously the notion that quantity of caloric may be distinct from the quantity of energy associated with it.

If we take the transfer of motive power (heat energy) associated with a quantity of caloric transferred between two bodies of equal temperature $T$ to be an extensive quantity (so that doubling the quantity of caloric transferred at that temperature doubles the transfer of motive power), then the motive power, $Q$, of a quantity $C$ of caloric at temperature $T$ will be given by,

$$
Q=C F(T)
$$

where $F(T)$ is a function of temperature. If we have a process in which a quantity $C$ of caloric is withdrawn from a reservoir at temperature $T_{1}$, with energy content $Q_{1}=C F\left(T_{1}\right)$, followed by cooling of the working substance to a lower temperature $T_{2}$ and deposit of the same quantity $C$ of caloric, with energy content $Q_{2}=C F\left(T_{2}\right)$, into a reservoir at temperature $T_{2}$, we must have

$$
\frac{F\left(T_{1}\right)}{F\left(T_{2}\right)}=\frac{Q_{1}}{Q_{2}},
$$

and, for the work developed by the engine,

$$
W=C\left(F\left(T_{1}\right)-F\left(T_{2}\right)\right) .
$$

The function $F$ that appears in (3) will, of course, depend on the temperature scale used. It was Kelvin's insight to realize that the fact that the maximum achievable efficiency of a heat engine operating between two reservoirs at temperatures $T_{1}$ and $T_{2}$ depends only on the 
temperatures can be used to define a natural, or absolute, temperature scale. We can define the ratio of the absolute temperature of the colder bath to the temperature of the hotter bath by

$$
\frac{T_{2}}{T_{1}}=1-\eta=\frac{Q_{2}}{Q_{1}}
$$

where $\eta$ is the efficiency of a reversible engine operating in a cycle between the two reservoirs, as defined in (2), and $Q_{1}$ and $Q_{2}$ are the quantities of heat energy withdrawn from, and deposited into, the hotter and colder reservoirs by the reversible engine. By Carnot's principle, it does not matter what reversible engine is considered.

When temperature is measured on the absolute scale, the function $F$ appearing in equation (3) takes a particularly simple form.

$$
F(T) \propto T,
$$

which gives us, for the heat energy $Q$ associated with a quantity $C$ of caloric transferred at temperature $T$,

$$
Q \propto C T .
$$

Thus, the work developed by a fall of a quantity $C$ of caloric from a temperature $T_{1}$ to a lower temperature $T_{2}$ is

$$
W \propto C\left(T_{1}-T_{2}\right)
$$

We can choose units for $C$, the quantity of caloric, that make the proportionality constant equal to unity. Thus we have that, when a quantity $C$ of caloric is transferred between bodies at absolute temperature $T$, there is an associated transfer of heat energy

$$
Q=C T
$$

All we have assumed, in order to obtain the result (10), is:

I. The heat energy associated with a quantity of caloric at a given temperature is an extensive quantity.

II. Caloric content is a function of the thermodynamic state of a system, so that, when a system undergoes a cyclic process, restoring at the end of the process its initial thermodynamic state, its initial caloric content is thereby restored. 
III. Conservation of energy; the work developed by the engine is equal to the difference between the heat energy extracted from the hotter reservoir and the heat energy deposited into the cooler reservoir.

These assumptions suffice to yield the relation (10), up to an irrelevant multiplicative constant. The relation can, of course, be inverted. When a quantity $Q$ of heat energy is transferred between two bodies at absolute temperature $T$, there is a transfer of a quantity of caloric equal to

$$
C=\frac{Q}{T}
$$

Given the assumptions I-III, we obtain (up to an irrelevant multiplicative constant) (11) as the quantity of caloric transferred between two bodies at equal temperatures, when a quantity $Q$ of heat energy is transferred via a thermodynamically reversible process.

Carnot himself derives the conclusion that $F(T)$ is proportional to $T$, for the special case of an ideal gas, and under the assumption that specific heat of a gas is independent of temperature and volume. ${ }^{14}$ There is a wrinkle, however. Carnot also takes the measure of a quantity of heat (caloric) to be its calorimetric measure; we may measure a quantity of heat by the temperature change it produces in some quantity of water, or by the quantity of ice that it will melt (see Carnot 1824, 15; 1890, 50; Magie 1899, 9). It is now taken to be a well-established fact that quantity of heat, as measured calorimetrically, is proportional to its energy content $Q$, with a proportionality independent of the substance in which the heat inheres and of the temperature of the bodies between which the heat is transferred. This would mean that, contrary to Carnot's assumption, the energy content (motive power) of a quantity of heat (measured calorimetrically) does not depend on temperature.

If we accept that the motive power (energy content) of a quantity (measured calorimetrically) of heat transferred in a thermodynamically reversible process is independent of temperature, and not (as Carnot thought) something that increases with temperature, then we have a decision to make. We can maintain that quantity of caloric is its calorimetric measure, and accept that caloric is not conserved in a Carnot cycle and that some of it is converted to mechanical work.

\footnotetext{
${ }^{14}$ This is in a footnote to the main text; see Carnot (1824, 73-79), Carnot (1890, 244251), Magie (1899, 39-42).
} 
Or we can take (11) as a theory-dependent measure of the quantity of caloric transferred when a quantity of heat energy is transferred between two bodies at the same temperature. This is a terminological choice. There is something to be said for the latter choice, as it helps us see why the assumption that caloric is conserved in a Carnot cycle does no harm; if quantity of caloric is given by (11), it is conserved in a Carnot cycle.

This definition of caloric content deals with transfers of caloric between bodies at equal temperature via thermodynamically reversible processes. As the caloric content of a system is taken to be a state function, the definition can be extended to changes in caloric content via irreversible transitions, provided that the initial and final states can be connected by some reversible process. Given a process that begins in a thermodynamic state $a$ and ends in a thermodynamic state $b$, we can consider a reversible process connecting the two states (it follows from the second law of thermodynamics that it won't matter which reversible process we consider), and apply the definition (11).

This has the consequence that total quantity of caloric in all bodies involved in a process is conserved only in thermodynamically reversible processes. In irreversible processes, caloric is generated. This may seem to some readers to strain the notion of caloric beyond the breaking point. I can only say, in defense of this locution, that at least one researcher, Kelvin himself, was willing to countenance the possibility of generating caloric, in his initial reaction to Joule's experiments. In a footnote appended to the word "impossible" in the above-quoted passage, he writes

* This opinion [of the impossibility of conversion of heat into mechanical effect] seems to be very nearly universally held by those who have written on the subject. A contrary opinion however has been advocated by Mr Joule of Manchester ; some very remarkable discoveries which he has made with reference to the generation of heat by the friction of fluids in motion, and some known experiments with magneto-electric machines, seeming to indicate an actual conversion of mechanical effect into caloric. No experiment however is adduced in which the converse operation is exhibited; but it must be confessed that as yet much is involved in mystery with reference to these fundamental questions of natural philosophy (Thomson 1848, 68; 1882, 102). 
On this view, caloric may be generated by expenditure of mechanical work, but, once generated, cannot be destroyed. Acceptance of this view requires that one take (11) as correctly furnishing the quantity of caloric associated with transfer of heat energy $Q$ at absolute temperature $T$.

The traditional account of the shift from caloric theory to the kinetic theory of heat might lead one to expect the quantity that we have been calling caloric content, defined by (11), to play no role in the theorizing of the kinetic theorists. An unsubtle wielding of the traditional realist response to the pessimistic meta-induction might involve an attempt to argue that quantity of caloric $C$, so defined, is a wheel turning idly in the theory, with all the essential work done by the quantity of heat energy $Q$. As the astute reader has already realized, nothing could be further from the truth!

The quantity, $Q / T$, was recognized by the kinetic theorists who were developing the nascent science of thermodynamics as a significant quantity. Clausius (1854) designated it the equivalence-value (Aequivalenzwerth) of the heat transferred. By 1865 he had decided that it required a more dignified name.

If we look for a designation for $S$, one might, in analogy to saying that the quantity $U$ is the body's heat and work content, say of the quantity $S$ that it is the transformational content of the body. But since I think it better to take the names of such important scientific quantities from ancient languages, so that they can be used unchanged in all modern languages, I propose that $S$ be called according to Greek words, $\dot{\eta} \tau \rho \omega \pi \dot{\eta}$, transformation, the entropy of the body. The word entropy was deliberately formed to be as similar as possible to the word energy, because the two magnitudes which are to be named by these words are so closely related to one another in their physical meanings, that a certain similarity in the designation seems to me to be appropriate (from Clausius 1865, 390; cf. Clausius $1867 \mathrm{c}, 357) .{ }^{15}$

\footnotetext{
${ }^{15}$ Sucht man für $S$ einen bezeichnenden Namen, so könnte man, ähnlich wie von der Grösse $U$ gesagt ist, sie sei der Wärme- und Werkinhalt des Körpers, von der Grösse sagen, sie sei der Verwandlungsinhalt des Körpers. Da ich es aber für besser halte, die Namen derartiger für die Wissenschaft wichtiger Grössen aus den alten Sprachen zu entnehmen, damit sie unverändert in allen neuen Sprachen angewandt werden können, so schlage ich vor, die Grösse $S$ nach dem griechischen Worte $\dot{\eta} \tau p \omega \pi \grave{\eta}$, die Verwandlung, die Entropie des
} 
In my alternate history, Clausius' neologism fails to catch on, and, in deference to tradition (or perhaps the principle that there is no point in inventing a new word when there is a perfectly serviceable readymade one), the word caloric is used for the Aequivalenzwerth of heat. The inhabitants of this alternate world take, as one formulation of the second law of thermodynamics, the principle that caloric, once generated, is never destroyed. They do not, of course, agree with Carnot in all respects. They don't think of caloric as a substance, but they rightly regard this a completely inessential aspect of the theoretical shift from Carnot of 1824 to their own. Their chief substantive difference with Carnot 1824 has to do with the conservation of caloric, but they acknowledge that, though Carnot was mistaken in thinking that the total quantity of caloric is constant, this does not affect his reasoning because quantity of caloric is conserved in thermodynamically reversible processes. Whether the caloric content of a body is thought of as the quantity of some substance contained in the body or is realized in some more subtle manner they rightly - and in agreement with Lavoisier ${ }^{16}$ — regard as irrelevant to any serious point of physical theory.

I am not the first to have thought along these lines; the same proposal, arrived at by much the same reasoning, was presented by Hugh Longbourne Callendar, in his Presidential Address to the Physical Society of London, on February 10, 1911. Callendar writes,

it was inevitable that caloric would make its reappearance sooner or later in the mechanical theory. It first appears, disguised as a triple integral, in Kelvin's solution ("Phil. Mag." IV., p. 305, 1852) of the problem of finding the work attainable from an unequally heated body. The solution (as corrected later) is equivalent to the statement that the total quantity of caloric remains constant when the equalisation of temperature is effected by means of reversible engines. Caloric reappears next as the "thermodynamic function" of Rankine, and as the "equivalence-value of a transformation" of Clausius ("Pogg. Ann.," XCIII., p. 497, 1854). Finally, in 1865, when its importance was more fully recog-

Körpers zu nennen. Das Wort Entropie habe ich absichtlich dem Wort Energie möglichst ähnlich gebildet, denn die beiden Grössen, welche durch diese Worte benannt werden sollen, sind ihren physikalischen Bedeutungen nach einander so nahe verwandt, dass eine gewisse Gleichartigkeit in der Benennung mir zweckmässig zu sein scheint.

${ }^{16}$ See quotation in footnote 13 , above. 
nised, Clausius ("Pogg. Ann.," CXXV., p. 390) gave it the name of "entropy," and defined it as the integral of $d Q / T$. This definition depends on the calorimetric or energy measure of heat, and obscures the fact that the caloric measure of heat follows directly from Carnot's principle, and may be made independent of the calorimetric measure. No one at that time appears to have appreciated Carnot's solution, or to have realised that entropy was merely caloric under another name. In justice to Carnot, it should be called caloric, and should be defined directly by means of his equation $W=A Q\left(T-T_{0}\right) .{ }^{17}$ This method of procedure appears to be justifiable both logically and historically, and leads to a more practical and definite conception of entropy or caloric as the true measure of a quantity of heat as opposed to a quantity of thermal energy (Callendar, 1910, 178).

The viability of interpreting Carnot's quantity of caloric as entropy was the subject of an exchange in the pages of the American Journal of Physics between Victor Kuhn La Mer and Thomas S. Kuhn (La Mer 1954, Kuhn 1955a, La Mer 1955, Kuhn 1955b). Unfortunately, much of the discussion revolved around La Mer's hyperbolic suggestion that an interpretation of Carnot consistent with the modern view can be obtained by consistently translating his "calorique" as "entropy" and "chaleur" as "heat" (Kuhn is correct that Carnot does not consistently distinguish calorique and chaleur in the manner required for this translation to go through).

\section{A matter of substance?}

In my alternate history, we still speak of the ether, but, with Einstein, regard it as synonymous with space devoid of ponderable matter. We speak of caloric, but we understand what our German colleagues mean when they occasionally use Clausius' neologism Entropie. This is not to say that our conception of the ether is to be identified with any of the various ideas of the ether that were employed in 19th-century optical and electromagnetic theory, or to say that our conception of

\footnotetext{
${ }^{17}$ Callendar's $Q$ is quantity of caloric, which is our $C$. In this equation, $W$ is the work obtained from a quantity of caloric via a fall of temperature from $T$ to $T_{0}$. This equation is, therefore, equivalent to our eq. (9).
} 
caloric is to be identified with Lavoisier's or Carnot's or any 18thor 19th-century writer. Lavoisier's conception of caloric is predicated on a conception of gases that extends the macroscopic quiescence of a gas in equilibrium to the molecular level; on Lavoisier's view, the molecules of a gas remain at constant distances from each other, and the role of caloric is to keep them that way, overcoming their natural attraction for each other. For Lavoisier, caloric is "the repulsive cause, whatever that may be, which separates the particles of matter from each other." We, on the other hand, reject the presupposition of this; we don't think that the molecules of a gas are kept separated from each other, except on average.

To some readers, no doubt, there will seem to be a deep gulf between the older notions of caloric and the one suggested here (which is compatible with the kinetic theory of heat), and between the older notions of ether and the one suggested here (which is compatible with the special and general theories of relativity), to the extent that they will balk at extending the use of the terms in this way. If we attempt to articulate the nature of this supposed gulf, there is, it seems, a natural and simple way to characterize it. On the older conceptions, caloric was conceived of as a substance. Adoption of the kinetic theory of heat requires rejection of caloric as a substance. The quantity that, in this context, may be called either quantity of caloric or entropy, indicates, not the quantity of some substance that a body contains, but rather, something about the body's internal configuration. Similarly, ether, if Einstein's suggestion is to be followed, is not, as Fresnel and Maxwell and Lorentz conceived it, an all-pervasive substance.

I must admit I share this intuition. ${ }^{18}$ We should ask whether this intuition can form the basis of a considered judgment. If we express the difference between caloric, as conceived of by Lavoisier, and entropy, as we conceive it, by saying that the latter is not a substance, what conception of substance are we employing to make this judgment?

One reason that this is a serious question is that those who were converted to the kinetic theory of heat by experiments involving the generation of heat by mechanical means employed inferences that we might not be willing to endorse. We repeatedly find rejections of the theory of heat as a substance based on a presupposition that, if heat is a substance, its quantity is conserved. So, for example, Count Rumford,

\footnotetext{
${ }^{18}$ When I say we have an intuition, I hope it is clear that I do not mean to indicate a source of a priori knowledge, but rather, to flag a potential obstacle to clear thinking.
} 
What is heat?-Is there any such thing as an igneous fluid?Is there any thing that can with propriety be called caloric?

... in reasoning on this subject, we must not forget to consider that most remarkable circumstance, that the source of the heat generated by friction, in these experiments, appeared evidently to be inexhaustible.

It is hardly necessary to add, that any thing which any insulated body, or system of bodies, can continue to furnish without limitation, cannot possibly be a material substance: and it appears to me to be extremely difficult, if not quite impossible, to form any distinct idea of any thing, capable of being excited, and communicated, in the manner the heat was excited and communicated in these experiments, except it be мотіоN (Thompson, 1798, 98-99).

If it is a necessary condition for something to be a material substance that it cannot be generated in unlimited quantities, given unlimited quantities of mechanical energy, then, by the lights of 20th-century physics, there are no material substances.

The basic picture guiding most of the 18th- and 19th- century discussions was this. The world is composed of a finite number of simple substances, whose quantity was fixed at the moment of creation and will persist until the end of days. Composites consist of arrangements of these simple substances, and all change consists of changes in these arrangements. This is a picture that has been rejected by 20th-century physics. One option we have is to say that we do not think that entropy is a substance in the sense meant by caloric theorists because we do not think that there are any substances in that sense. But this makes the thesis trivial. I leave it an open question, for the reader to consider, as to what, if any, notion of substance might serve to underwrite the thesis without trivializing it.

\section{Conclusion}

If one examines the history of electromagnetic theory or of the theory of heat prior to the twentieth century, one thing that presents itself most forcefully is that there was never a settled and agreedupon body of theory. At any time there were multiple theories, none of which could be taken as a final, completely adequate theory. And, as concepts such as "caloric" and "ether" are theory-dependent, there 
was never a settled conception of these. These were times of theories and concepts in flux. For this reason, I do not regard it as a well-posed question to ask whether we should take the words "caloric" or "ether", as used by 19th-century authors, as referring. We should decide what we believe the world to be like, and, insofar as this conception differs from 19th-century conceptions, we have a choice of whether to continue to employ older terminology or adopt new coinages.

As I said in the outset, there is a tendency to be beguiled by contingent matters of retention and non-retention of terms. The fact that Lorentz continued to speak of "ether" obscures the revolutionary nature of the move that he made, a crucial one for the creation of the subject that, in 20th-century textbooks, solidified as "classical electrodynamics." The fact that Einstein in 1905 rejected the ether as superfluous obscures the extent of the continuity between his thought and Lorentz's. Einstein in later years tried to remedy this fault, with (sadly, in my opinion) little effect on the way we talk about the transition from 19th- to 20th-century electromagnetic theory.

The fact that converts to the kinetic theory of heat rejected the use of the word "caloric" (which had previously been taken as more or less synonymous with "heat") while retaining the word "heat" is also, in my opinion, unfortunate. As a result of this, we continue to talk of heat flow between bodies, and, then, we have to explain to our students that we don't thereby mean a increase in heat content of the receiving body and decrease of heat content of the transmitting body, and that it makes no sense to speak of the heat content of a body, as energy that enters a body as heat may leave it as work, and vice versa. Though heat content of a body is not a function of its thermodynamic state, caloric content, as we have defined it, is a state function. We would be much better off, and have fewer occasions to explain to our students that we don't mean what our words suggest, if we had retained the term caloric and abandoned the word heat.

\section{Appendix: Boundary conditions for electric and magnetic fields}

The demonstration that Fresnel's laws of reflection and refraction follow from Maxwell's equations has become a textbook staple; see, e.g. Jackson $(1962, \S 7.5)$. The boundary conditions required are continuity of the normal components of $\mathbf{D}$ and $\mathbf{B}$ at the boundary, and of 
the tangential components of $\mathbf{E}$ and $\mathbf{H}$. The continuity condition for the normal component of $\mathbf{B}$ and the tangential component of $\mathbf{E}$ are consequences of Maxwell's equations. Continuity of the normal component of $\mathbf{D}$ and of the tangential components of $\mathbf{H}$ are equivalent to the absence of surface charge and surface current, respectively, at the boundary. In this appendix is provided the proof of these assertions. This demonstration can be found in many textbooks of electromagnetism; see, e.g. Jackson (1962): $\S 4.4$ for the electric conditions, and $\S 5.9$ for the magnetic.

We assume the macroscopic version of Maxwell's equations, and adopt Gaussian units. Maxwell's equations, in differential form, are,

$$
\begin{array}{r}
\nabla \cdot \mathbf{D}=4 \pi \rho \\
\nabla \times \mathbf{H}=\frac{1}{c}\left(4 \pi \mathbf{J}+\frac{\partial \mathbf{D}}{\partial t}\right) \\
\nabla \cdot \mathbf{B}=0 \\
\nabla \times \mathbf{E}+\frac{1}{c} \frac{\partial \mathbf{B}}{\partial t}=0 .
\end{array}
$$

For our purpose the integral form of the equations is most convenient. For (16) and (18), let $V$ be the volume bounded by a closed surface $S$, and let $d \mathbf{S}$ be $\hat{\mathbf{n}} d a$, where $d a$ is a surface element and $\hat{\mathbf{n}}$ is the outward-pointing unit normal vector. For (17) and (19), let $C$ be a closed curve, and let $S$ be the surface bounded by this curve, and let $d \mathbf{S}=\hat{\mathbf{n}} d a$, with $\hat{\mathbf{n}}$ a normal to the surface, chosen so that the traversal of the curve is counterclockwise, when viewed from the side $\hat{\mathbf{n}}$ picks out as positive. Then we have,

$$
\begin{array}{r}
\int_{S} \mathbf{D} \cdot d \mathbf{S}=4 \pi \int_{V} \rho d V ; \\
\int_{C} \mathbf{H} \cdot d \mathbf{l}=\frac{1}{c} \int_{S}\left(4 \pi \mathbf{J}+\frac{\partial \mathbf{D}}{\partial t}\right) \cdot d \mathbf{S} \\
\int_{S} \mathbf{B} \cdot d \mathbf{S}=0 ; \\
\int_{C} \mathbf{E} \cdot d \mathbf{l}=-\frac{1}{c} \frac{d}{d t} \int_{S} \mathbf{B} \cdot d \mathbf{S} .
\end{array}
$$

Consider two regions, 1 , and 2, containing a homogenous isotropic substances, of differing dielectric constants and magnetic susceptibilities. Let $S$ be the boundary surface between them (assumed smooth). Consider a small region of this surface, sufficiently small that we may 
treat it as planar. Choose coordinates so that the $z$-axis is normal to the surface $S$, pointing from region 1 to region 2 .

Consider a region whose "top" and "bottom" consist of surfaces of area $A$, parallel to the boundary surface $S$, with the top within region 2 and the bottom within region 1 . The sides are of length $\varepsilon$, and intersect $S$ at right angles. Let $D_{2 z}-D_{1 z}$ be the discontinuity (if any) of the $z$-component of $\mathbf{D}$ at the boundary.

If we take $\varepsilon$ sufficiently small as to be negligible, then the charge inside our region is $\sigma A$, where $\sigma$ is the surface charge density at the boundary, and the integral of $\mathbf{D} \cdot d \mathbf{S}$ over the surface of our region is $\left(D_{2 z}-D_{1 z}\right) A$. By $(16)$,

$$
\left(D_{2 z}-D_{1 z}\right) A=4 \pi \sigma A
$$

or,

$$
\left(D_{2 z}-D_{1 z}\right)=4 \pi \sigma
$$

Another (coordinate-independent) way of putting this is: If $\hat{\mathbf{n}}$ is the unit normal at the surface,

$$
\hat{\mathbf{n}} \cdot\left(\mathbf{D}_{2}-\mathbf{D}_{1}\right)=4 \pi \sigma .
$$

The discontinuity of the normal component of $\mathbf{D}$ at the boundary is proportional to the surface charge density at the boundary. Using the same construction, (18) yields continuity of the normal component of B at the boundary.

$$
\hat{\mathbf{n}} \cdot\left(\mathbf{B}_{2}-\mathbf{B}_{1}\right)=0 .
$$

Now consider a small oriented closed curve, consisting of a segment of length $L$ parallel to the surface, and oriented in the $x$-direction, followed by a segment of length $\varepsilon$ in the positive $z$-direction that crosses the boundary, a segment of length $L$ in the negative $x$-direction, and a segment of length $\varepsilon$ in the negative $z$-direction, that closes the curve. Let $\mathbf{K}$ be the surface current density at the boundary. Again, taking $\varepsilon$ to be small enough that contributions from the sides are negligible, the integral of $\mathbf{H} \cdot d \mathbf{l}$ around this loop is $\left(H_{1 x}-H_{2 x}\right) L$, and the current through the surface bounded by the loop is $-K_{y} L$. As the total area of the surface bounded by the loop can be made arbitrarily small by taking $\varepsilon$ small, the contribution of the displacement-current term to the right-hand right-hand-side of (17) can also be neglected, and we have

$$
\left(H_{1 x}-H_{2 x}\right) L=\frac{4 \pi}{c} K_{y} L
$$


or,

$$
H_{1 x}-H_{2 x}=\frac{4 \pi}{c} K_{y} .
$$

If we now consider a loop with sides oriented in the $y$-direction, we get

$$
H_{1 y}-H_{2 y}=-\frac{4 \pi}{c} K_{x} .
$$

Thus, tangential components of $\mathbf{H}$ satisfy,

$$
\hat{\mathbf{n}} \times\left(\mathbf{H}_{2}-\mathbf{H}_{1}\right)=\frac{4 \pi}{c} \mathbf{K},
$$

where $\hat{\mathbf{n}}$ is a unit vector normal to the boundary surface. The same construction applied to (19) yields continuity of the tangential components of $\mathbf{E}$.

$$
\hat{\mathbf{n}} \times\left(\mathbf{E}_{2}-\mathbf{E}_{1}\right)=0 .
$$

\section{Acknowledgments}

Hearty thanks for their helpful comments are extended to the audience at the conference, The Philosophy of Howard Stein, and Its Contemporary Interest (June 9-11, 2017, Chicago), and to the members of the Southwestern Ontario Philosophy of Physics discussion group. And, of course, this paper would not have come about were it not for the groundbreaking work of Howard Stein. 


\section{References}

Bromberg, J. (1968). Maxwell's electrostatics. American Journal of Physics 36, 142-151.

Buchwald, J. Z. (1985). From Maxwell to Microphysics: Aspects of Electromagnetic Theory in the Last Quarter of the Nineteenth Century. Chicago: The University of Chicago Press.

Callendar, H. L. (1910). The caloric theory of heat and Carnot's principle. Proceedings of the Royal Society of London 23, 153-189.

Carnot, S. (1824). Réflexions sur la Puissance Motrice du Feu et sur les Machines Propres a Déveloper Cette Puissance. Paris: Bachelier. Translated in Carnot (1890), and in Magie (1899).

Carnot, S. (1890). Reflections on the Motive Power of Fire, and on Machines Fitted to Develop That Power. London: MacMillan \& Co. Translation of Carnot (1824).

Clausius, R. (1854). Ueber eine veränderte Form des zweiten Hauptsatzes der mechanischen Wärmetheorie. Annalen der Physik 93, 481-506. Reprinted in Clausius (1864, 127-154); English translation in Clausius (1856), and in Clausius (1867b).

Clausius, R. (1856). On a modified form of the second fundamental theorem in the mechanical theory of heat. The London, Edinburgh, and Dublin Philosophical Magazine and Journal of Science 12, 8188. English translation of Clausius (1854).

Clausius, R. (1864). Abhandlungen über die mechanische Wärmetheorie, Volume 1. Braunschweig: Friedrich Vieweg und Sohn.

Clausius, R. (1865). Ueber verschiedene für die Anwendung bequeme Formen der Hauptgleichungen der mechanischen Wärmetheorie. Annalen der Physik 125, 353-400. Reprinted in Clausius (1867a, 1-44). English translation in Clausius (1867b).

Clausius, R. (1867a). Abhandlungen über die mechanische Wärmetheorie, Volume 2. Braunschweig: Friedrich Vieweg und Sohn.

Clausius, R. (1867b). The Mechanical Theory of Heat, with its Applications to the Steam Engine, and to the Physical Properties of 
Bodies. London: John van Voorst. English translation, with one additional paper, of Clausius (1864).

Clausius, R. (1867c). On several convenient forms of the fundamental equations of the mechanical theory of heat. In Clausius (1867b), 327-374. English translation of Clausius (1865).

Darrigol, O. (2000). Electrodynamics from Ampère to Einstein. Oxford: Oxford University Press.

Einstein, A. (1905). Zur Elektrodynamik bewegter Körper. Annalen der Physik 17, 891-921.

Einstein, A. (1920). Aether und Relativitätstheorie. Berlin: Julius Springer.

Einstein, A. (1922). Ether and the theory of relativity. In Sidelights on Relativity, 3-24. London: Methuen \& Co. Ltd. Translation, by G. B. Jeffery and W. Perrett, of Einstein (1920).

Einstein, A. (1934). Das Raum-, Äther- und Feld-Problem der Physik. In C. Seelig (Ed.), Mein Weltbild, 229-248. Amsterdam: Querido.

Einstein, A. (1954). The problem of space, ether, and the field in physics. In Ideas and Opinions, 276-285. New York: Crown Publishers Inc. English translation, by Sonia Bargmann, of Einstein (1934).

Einstein, A. (1989). On the electrodynamics of moving bodies. In The Collected Papers of Albert Einstein, Volume 2: The Swiss Years: Writings, 1900-1909 (English translation supplement). Princeton: Princeton University Press. Translation, by Anna Beck, of Einstein (1905).

Faraday, M. (1846). Experimental researches in electricity.Nineteenth series. Philosophical Transactions of the Royal Society of London 136, 1-20.

Fitzgerald, G. F. (1880). On the electromagnetic theory of the reflection and refraction of light. Philosophical Transactions of the Royal Society of London 171, 691-711. 
Harman, P. M. (Ed.) (1995). The Scientific Letters and Papers of James Clerk Maxwell, Volume II: 1862-1873. Cambridge: Cambridge University Press.

Harman, P. M. (Ed.) (2002). The Scientific Letters and Papers of James Clerk Maxwell, Volume III: 1874-1879. Cambridge: Cambridge University Press.

Heaviside, O. (1892). Electrical Papers, Volume I. London: MacMillan and Co.

Helmholtz, H. v. (1870). Ueber die Theorie der Elektrodynamik. Erste Abhandlung: Ueber die Bewegungsgleichungen der Elektricität für ruhende leitende Körper. Journal für die reine und angewandte Mathematik 72, 57-129. Reprinted in Helmholtz (1882, 545-628).

Helmholtz, H. v. (1882). Wissenschaftliche Abhandlungen. Erster Band. Leipzig: Johann Ambrosius Barth.

Hirosige, T. (1969). Origins of Lorentz' theory of electrons and the concept of the electromagnetic field. Historical Studies in the Physical Sciences 1, 151-209.

Jackson, J. D. (1962). Classical Electrodynamics. New York: John Wiley \& Sons.

Kuhn, T. S. (1955a). Carnot's version of "Carnot's cycle". The American Journal of Physics 23, 91-95.

Kuhn, T. S. (1955b). Carnot's version of "Carnot's cycle". The American Journal of Physics 23, 387-389.

La Mer, V. K. (1954). Some current misinterpretations of N. L. Sadi Carnot's memoir and cycle. The American Journal of Physics 22, $20-27$.

La Mer, V. K. (1955). Some current misinterpretations of N. L. Sadi Carnot's memoir and cycle. II. The American Journal of Physics 23, 95-102.

Lavoisier, A. (1789). Traité Élémentaire de Chimie. Paris: Cluchet.

Lavoisier, A. (1790). Elements of Chemistry. Edinburgh: William Creech. English translation, by Robert Kerr, of Lavoisier (1789). 
Lorentz, H. A. (1875). Over de Theorie der Terugkaatsing en Breking van het Licht. Arnhem: K. van der Zande. Reprinted, with French translation, in Lorentz (1935).

Lorentz, H. A. (1892). La Théorie Électromagnétique de Maxwell et son Application aux Corps Mouvants. Leiden: Brill.

Lorentz, H. A. (1935). Collected Papers, Volume I. The Hague: Martinus Nijhoff.

Lorentz, H. A. (1997). On the Theory of the Reflection and Refraction of Light. Amsterdam: Rodopi. English translation, by Nancy J. Nersessian and H. Floris Cohen, of Lorentz (1875).

MacCullagh, J. (1846). An essay towards a dynamical theory of crystalline reflexion and refraction. The Transactions of the Royal Irish Academy 21, 17-50.

Magie, W. F. (Ed.) (1899). The Second Law of Thermodynamics: Memoires by Carnot, Clausius, and Thomson. New York and London: Harper \& Brothers.

Maxwell, J. C. (1858). On Faraday's lines of force. Transactions of the Cambridge Philosophical Society 10, 27-83. Reprinted in Niven (1890, 155-229).

Maxwell, J. C. (1861). On physical lines of force I, II. Philosophical Magazine 21, 161-175, 281-291. Reprinted in Niven (1890, 451488).

Maxwell, J. C. (1862). On physical lines of force III, IV. Philosophical Magazine 23, 12-24, 85-95. Reprinted in Niven (1890, 489-513).

Maxwell, J. C. (1865). A dynamical theory of the electromagnetic field. Philosophical Transactions of the Royal Society of London 155, 459 512. Reprinted in Niven $(1890,526-597)$.

Maxwell, J. C. (1873a). A Treatise on Electricity and Magnetism, Vol. I. Oxford: The Clarendon Press.

Maxwell, J. C. (1873b). A Treatise on Electricity and Magnetism, Vol. II. Oxford: The Clarendon Press. 
Maxwell, J. C. (1879). Report on a paper by George Francis Fitzgerald on the electromagnetic theory of the reflection and refraction of light. In Harman (2002), 751-755.

Maxwell, J. C. (1881). A Treatise on Electricity and Magnetism, Vol. I (2nd ed.). Oxford: The Clarendon Press.

Niven, W. D. (Ed.) (1890). The Scientific Papers of James Clerk Maxwell, Volume One. Cambridge: Cambridge University Press.

Poincaré, H. (1890). Électricité et Optique I: Les Théories de Maxwell et la théorie électromagnétique de la lumière. Paris: Georges Carré.

Saatsi, J. (2005). Reconsidering the Fresnel-Maxwell theory shift: how the realist can have her cake and EAT it too. Studies in History and Philosophy of Science 36, 509-538.

Siegel, D. M. (1991). Innovation in Maxwell's Electromagnetic Theory: Molecular vortices, displacement current, and light. Cambridge: Cambridge University Press.

Stanford, P. K. (2015). "Atoms exist" is probably true, and other facts that should not comfort scientific realists. The Journal of Philosophy 112, 397-416.

Stein, H. (1978). After the Baltimore lectures: Some philosophical reflections on the subsequent development of physics. In R. Kargon and P. Achinstein (Eds.), Kelvin's Baltimore Lectures and Modern Theoretical Physics: Historical and Philosophical Perspectives, 375-398. Cambridge: The MIT Press.

Stein, H. (1981). 'Subtler forms of matter' in the period following Maxwell. In G. N. Cantor and M. J. S. Hodge (Eds.), Conceptions of Ether: Studies in the history of ether theories 1740-1900, 309340. Cambridge: Cambridge University Press.

Stein, H. (1989). Yes, but...some skeptical remarks on realism and anti-realism. Dialectica 43, 47-65.

Thompson, B. (1798). An inquiry concerning the source of the heat which is excited by friction. Philosophical Transactions of the Royal Society of London 88, 80-102. 
Thomson, W. (1847). On a mechanical representation of electric, magnetic, and galvanic forces. The Cambridge and Dublim Mathematical Journal 2, 61-64. Reprinted in Thomson (1882, 76-80).

Thomson, W. (1848). On an absolute thermometric scale founded on Carnot's theory of the motive power of heat, and calculated from Regnault's observations. Proceedings of the Cambridge Philosophical Society 1(5), 66-71. Reprinted in Thomson (1882, 100-106).

Thomson, W. (1849). An account of Carnot's theory of the motive power of heat; with numerical results deduced from Regnault's experiments on steam. Transactions of the Royal Society of Edinburgh 16, 541-574. Reprinted in Thomson (1882, 113-155).

Thomson, W. (1882). Mathematical and Physical Papers, Volume I. Cambridge: Cambridge University Press.

Worrall, J. (1989). Structural realism: The best of both worlds? Dialectica 43, 99-124.

Worrall, J. (1994). How to remain (reasonably) optimistic: Scientific realism and the "luminiferous ether". In PSA: Proceedings of the Biennial Meeting of the Philosophy of Science Association, Volume One: Contributed Papers (1994), 334-342. 